\title{
Competitive Aerobics Athlete Body Function Comparative Analysis and Comprehensive Evaluation Mode Application
}

\author{
Liping Wang*
}

Institute of Physical Education, Hunan University Of Science and Engineering, Yongzhou 425199, Hunan, China

\begin{abstract}
The paper studies competitive aerobics athletes body function, after making comprehensive evaluation on one group of competitive aerobics athletes body functions, obtained result is competitive aerobics athletes body function belongs to good level, by making comprehensive evaluation on competitive aerobics athletes body function, it well verifies fuzzy mathematics adaptability and effectiveness, and utilizes performance quantization method, to further research, the paper carries out concrete analysis on three competitive aerobics athletes body function, finally gets the three competitive aerobics athletes body function total performances rank $s_{1}<s_{3}<s_{2}$, and finally proposes methods to promote competitive aerobics athletes body function, and provides theoretical supports for future researching on competitive aerobics athletes body function.
\end{abstract}

Keywords: Body function, comparative analysis, competitive aerobics, fuzzy comprehensive evaluation.

\section{INTRODUCTION}

With aerobics rapidly popularization, in recent years, it has been favored by broad young people, regarding aerobics competitions are also emerging in endlessly, but to promote its comprehensive performances in competitions, it needs to go deeper analysis and research on aerobics athletes body function level.

Regarding aerobics aspect research, formers have made many efforts and obtained abundant achievements, such as : in aerobics optional course students learning evaluation criterion construction with Hebei province as an example, Dong Hui proposed that current Hebei province aerobics students' learning status was not going well, and defined evaluation system, from which it contained emotion attitudes, techniques technology and other aspects that provided evidence for defining standardized evaluation [1-3]; in aerobics athlete ability structure correlation analysis, Chang Sheng according to aerobics features, applies questionnaire survey, documents literature and other methods to analyze factors that affected athletes' ability, finally got that aerobics would develop towards high completion type, high innovation type, high artistry type and high difficulty type, all kinds of technical motions would be more coordinated till arrive at perfect [4-7].

The paper just on the basis of formers research, makes further analysis and researches on competitive athletes' body function, by applying questionnaire survey, mathematical statistics, fuzzy mathematics method and else multiple methods to analyze, and finally gets relative reasonable results, and meanwhile also proves the model rationality and effectiveness.

\section{FUZZY MATHEMATICS-BASED COMPREHEN- SIVE EVALUATION MODEL THEORETICAL ANALYSES}

Competitive aerobics athlete body function suffers many kinds of factors influences, but these factors have fuzzy and uncertainty, apply previous method is difficult to make evaluation, we present fuzzy mathematical comprehensive evaluation model to more reasonable establish competitive aerobics athlete body function comprehensive evaluation system $[8,9]$. Make use of maximum membership (remark) and fuzzy linear transformation principle to construct fuzzy comprehensive evaluation such basic thought is the model relative theory, we consider evaluated things related multiple factors influences conditions are considering in case extreme fuzzy, so that realize some purpose of making relative reasonable comprehensive evaluation on another kind of things. So we utilize fuzzy mathematics to carry out comprehensive evaluation, its methods and steps are as following:

At first, it should define evaluated objects that is individual variable affected by $n$ pieces of factors, and its factor set is $u$, definition is:

$u=\left(u_{1}, u_{2}, u_{3}, \cdots, u_{n}\right)$

And regulate

$u_{i}(i=1,2,3, \cdots, n)$

Due to each variable weight is different, influences degrees are different to defined judgment grade, we assume its weight allocation is $a_{i}$, and:

$a_{i}=\left(a_{1}, a_{2}, a_{3}, \cdots a_{n}\right)$

Among them

2015 Bentham Open 
$a_{i}(i=1,2,3, \cdots, n)$

It is weight of formula (2), according to common sense, we know $a_{i} \geq 0$ and $\sum_{i=1}^{n} a_{i}=1$.

If every factor $a_{i}$ includes $m$ pieces of sub factors, its factor set is

$u_{i}=\left(u_{i, 1,} u_{i, 2}, u_{i, 3}, \cdots, u_{i, m}\right)$

Then corresponding weight value is:

$a_{i}=\left(a_{i, 1,} a_{i, 2}, a_{i, 3}, \cdots a_{i, m}\right)$

To $u_{i, j}$ weight value $a_{i}$, according to common sense, it is clear $a_{i, j} \geq 0$ and $\sum_{j=1}^{m} a_{i, j}=1$

Establish an evaluation indicator set

$v=\left(v_{1}, v_{2},{ }_{3}, \cdots, v_{s}\right)$

Corresponding evaluation objects can be divided into $S$ pieces of different grades, here, we let $v_{1}, v_{2},{ }_{3}, \cdots, v_{s}$ to be each merits evaluation degree from high to low, such as excellent, good, qualified, and unqualified so on.

After defining every factor $u_{i, j}$ evaluation indicator evaluation degree, it makes evaluation on factor $u_{i}$ fuzzy comprehensive evaluation model, we let $u_{i, j}(j=1,2,3, \cdots, m)$ to be $r_{i}=\left(a_{i, 1} a_{i, 2}, a_{i, 3}, \cdots a_{i, m}\right) *\left(r_{i, 1}\right)^{T}, i=1,2,3, \cdots n \quad$ fuzzy comprehensive evaluation set of evaluation indicators $v$ hypothesis.

It gets required comprehensive evaluation result by fuzzy matrix compound calculation,

That is

$b=a * r=\left(a_{1}, a_{2}, a_{3}, \cdots a_{n}\right) *\left(r_{1}, r_{2}, r_{3}, \cdots, r_{n}\right)^{T}$

$=\left(b_{1}, b_{2}, b_{3}, \cdots, b_{n}\right)$

From fuzzy set $b$, we can make use of maximum evaluation degree method to get a definite evaluation level. Because $B_{k}=\left\{B_{I}\right\}$, then $B_{K}$ final evaluation result level is $k$.

\section{FUZZY MATHEMATICAL COMPETITIVE AERO- BICS ATHLETE BODY FUNCTION COMPREHEN- SIVE EVALUATION}

By above, it is known $u=\left(u_{1}, u_{2}, \cdots, u_{n}\right), u_{1}$ is $u_{i}$ corresponding weight value, $u$ can be defined by investigation, experience statistic and other methods. Take one competitive aerobics athlete body function to make comprehensive evaluation, after that, we combine each kind of factor so that compose of comprehensive evaluation transformation matrix $r_{1}$.

Comprehensive evaluation $a_{1}$ $a_{11}=u * r_{11}, a_{12}=u * r_{12}, \cdots, a_{1 n}=u_{1 n} * r_{1 n}$

After that we can combine $a_{11}$ into matrix $r_{11}$

Comprehensive evaluation $a_{2}$

$a_{2}=u^{*} r_{2}$

Take intersection from above two comprehensive evaluations

Comprehensive evaluation scores

$$
b=a * r^{T}
$$

Among them, $r^{T}$ is $r$ transformation matrix, and $r$ is Table 1 scores' matrix form.

$\ominus$ Competitive aerobics athlete body function comprehensive evaluation contents are defined, as following Fig. (1) shows:

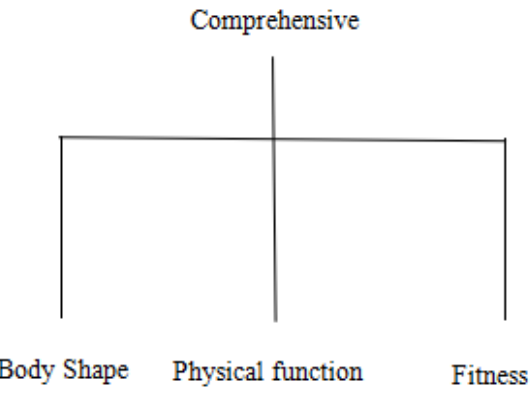

Fig. (1). Body Shape ordinary university college Competitive Aerobics Athletes learn the function of influencing factors Figure.

$\ominus$ Weight value layout

Competitive aerobics athlete comprehensive evaluation $(u)$ - competitive aerobics athlete body function $20 \%$ (0.2); competitive aerobics athlete body shape $20 \%(0.2)$; competitive aerobics athlete physical quality $60 \%(0.6)$

Competitive aerobics athlete body shape —upper limb length factor $50 \%(0.5)$, lower limb length factor $50 \%(0.5)$

Competitive aerobics athlete body function $\left(u_{1}\right)-$ motor function $60 \%(0.6)$; heart-lung function $20 \%(0.2)$; brain function $20 \%(0.2)$

Competitive aerobics athlete physical quality $\left(u_{2}\right)$ waist and abdomen factor $10 \%(0.1)$; jumping ability factor $5 \%(0.25)$; flexibility factor $50 \%(0.5)$; limbs movement factor $15 \%(0.15)$ 。

$\circledast$ We select one group of competitive aerobics athletes of them to carry out individual factor evaluation, its remarks codes are: $4,5,5,4,3,4,5,4,4$. In the following, we divide athletes comprehensive evaluation into two parts as following: 
Table 1. Parameters allocation.

\begin{tabular}{|c|c|c|c|c|c|}
\hline & Score & $\mathbf{2 0}$ & $\mathbf{4 0}$ & $\mathbf{8 0}$ & $\mathbf{1 0 0}$ \\
\hline \hline 1 & Well (first grade, excellent) & 0 & 0 & 0.25 & 0.75 \\
\hline 2 & Good (better than average, good) & 0 & 0 & 0.50 & 0.25 \\
\hline 3 & Normal (middle, qualified) & 0 & 0.25 & 0.25 & 0 \\
\hline 4 & Not good (middle, low grade) & 0.25 & $0 . .5$ & 0 & 0 \\
\hline 5 & Bad (lower grade, unqualified) & 0.75 & 0.25 & 0 \\
\hline
\end{tabular}

The group of athletes comprehensive evaluation one is:

Competitive aerobics athlete body function:

$a_{11}=u * r=\left[\begin{array}{ll}0.5 & 0.5\end{array}\right]$

Competitive aerobics athlete body shape:

$$
\begin{aligned}
a_{12} & =u_{2} * r_{12}=\left[\begin{array}{lll}
0.20 & 0.20 & 0.60
\end{array}\right] *\left[\begin{array}{ccccc}
0 & 0.25 & 0.50 & 0.25 & 0 \\
0 & 0 & 0.25 & 0.50 & 0.25 \\
0 & 0 & 0 & 0.25 & 0.75
\end{array}\right] \\
& =\left[\begin{array}{lllll}
0 & 0.05 & 0.15 & 0.3 & 0.5
\end{array}\right]
\end{aligned}
$$

Competitive aerobics athlete physical quality:

$$
a_{13}=u_{3} * r_{13}=\left[\begin{array}{llll}
0.15 & 0.50 & 0.25 & 0.10
\end{array}\right] *\left(\begin{array}{ccccc}
0 & 0 & 0.25 & 0.50 & 0.25 \\
0 & 0 & 0 & 0.25 & 0.75 \\
0 & 0 & 0 & 0.25 & 0.75 \\
0 & 0 & 0.25 & 0.50 & 0.25
\end{array}\right)
$$

$=\left[\begin{array}{lllll}0 & 0 & 0.0625 & 0.3125 & 0.625\end{array}\right]$

The group of athletes' comprehensive evaluation two is

$$
\begin{aligned}
a_{2} & =u * r_{2}=\left[\begin{array}{lll}
0.6 & 0.20 & 0.20
\end{array}\right] *\left(\begin{array}{ccccc}
0 & 0 & 0.0625 & 0.3125 & 0.625 \\
0 & 0.05 & 0.15 & 0.3 & 0.5 \\
0 & 0 & 0.25 & 0.5 & 0.25
\end{array}\right) \\
& =\left[\begin{array}{lllll}
0 & 0.01 & 0.1175 & 0.3475 & 0.525
\end{array}\right]
\end{aligned}
$$

The group of competitive aerobics athletes' comprehensive evaluation score is

$$
b=a_{2} * r^{T}=\left[\begin{array}{lllll}
0 & 0.01 & 0.1175 & 0.3475 & 0.525
\end{array}\right] *\left[\begin{array}{c}
20 \\
40 \\
60 \\
80 \\
100
\end{array}\right]
$$$$
=86.7
$$

By above, it is clear that the group of competitive aerobics athletes' competitive aerobics body function belongs to good level.

According to competitive aerobics features, it needs to establish a factor set about evaluation objects

$$
u=\left(u_{1}, u_{2}, u_{3}, \cdots, u_{n}\right)
$$

Secondly, according to competitive aerobics athlete body function, it establishes lateral split index, vertical split index, standing long jump, 30 seconds push-up, cross jumping test and others six items, corresponding factors use $u_{1}, u_{2}, u_{3}, \cdots, u_{6}$ to express

$$
u=\left(u_{1} u_{2}, u_{3}, \cdots, u_{6}\right)
$$

After that, divide competitive aerobics athletes body function performances successively as: excellent, good, qualified, bad these four grades to evaluate, so corresponding set is $v=\{$ excellent, good, qualified, $\operatorname{bad}\}=\left\{v_{1}, v_{2}, v_{3}, v_{4}\right\}$, we let $r_{i j}$ to be the $j$ factor the $i$ remark possibility extent.

For six aspect three competitive aerobics athletes body functions $(s)$, it carries out evaluation, by evaluation result, it can get:

$20 \% \quad(0.2)$ excellent, $23 \%$ good, $47 \%$ medium, $10 \%$ qualified and $0 \% \mathrm{bad}$, and assign values on them respectively as: $5,4,3,2,1$, then corresponding each grade weight can be got by calculation:

Excellent: $5 /(1+2+3+4+5)=0.33$

Good: $4 /(1+2+3+4+5)=0.27$

Medium: $3 /(1+2+3+4+5)=0.2$

Qualified: $2 /(1+2+3+4+5)=0.13$

Bad: $1 /(1+2+3+4+5)=0.07$

By above, it is clear corresponding weights are written into vector as:

$$
a=\left(a_{1}, a_{2}, \cdots, a_{6}\right)=(0.33,0.27,0.2,0.13,0.07)
$$

Then the thirty people to $S_{1}$ athlete six evaluation vectors are respectively :

$u_{1}$ evaluation vector is $0.230 .45,0.24,0.1,0.00$

$u_{2}$ evaluation vector is c0.06 0.11,0.36,0.31,0.17

$u_{3}$ evaluation vector is c0.20 0.36, 0.18,0.17,0.00

$u_{4}$ evaluation vector is c0.20 0.46,0.24,0.10,0.10

$u_{5}$ evaluation vector is c0.36 0.00,0.43,0.13,0.07

$u_{6}$ evaluation vector is c0.04 0.00,0.23,0.16,0.13

Through corresponding processing, we get competitive aerobics athletes body functions $s_{1}, s_{2}, s_{3}$ evaluation matrixes as: 


$$
\begin{aligned}
r_{1} & =\left(\begin{array}{llllll}
0.23 & 0.06 & 0.20 & 0.20 & 0.36 & 0.04 \\
0.45 & 0.11 & 0.36 & 0.46 & 0.00 & 0.00 \\
0.24 & 0.36 & 018 & 0.24 & 0.43 & 0.23 \\
0.10 & 0.31 & 0.17 & 0.10 & 0.13 & 0.16 \\
0.00 & 0.17 & 0.00 & 0.10 & 0.07 & 0.13
\end{array}\right) \\
r_{2} & =\left(\begin{array}{llllll}
0.03 & 0.51 & 0.20 & 0.30 & 0.40 & 0.07 \\
0.10 & 0.27 & 0.36 & 0.40 & 0.37 & 0.37 \\
0.47 & 0.13 & 018 & 0.17 & 0.00 & 0.40 \\
0.20 & 0.11 & 0.17 & 0.07 & 0.17 & 0.07 \\
0.20 & 0.00 & 0.00 & 0.07 & 0.07 & 0.10
\end{array}\right) \\
r_{3} & =\left(\begin{array}{llllll}
0.00 & 0.03 & 0.00 & 0.30 & 0.10 & 0.07 \\
0.07 & 0.20 & 0.20 & 0.30 & 0.17 & 0.07 \\
0.23 & 0.17 & 0.63 & 0.27 & 0.67 & 0.20 \\
0.17 & 0.57 & 0.13 & 0.07 & 0.03 & 0.57 \\
0.53 & 0.03 & 0.03 & 0.07 & 0.03 & 0.10
\end{array}\right)
\end{aligned}
$$

Then by fuzzy mathematical evaluation matrix, through corresponding linear transformation, respectively transform three competitive aerobics athletes body functions $s_{1}, s_{2}, s_{3}$ evaluation matrixes:

Athlete $S_{1}$ linear transformation is

$$
\begin{gathered}
b_{1}=\left(\begin{array}{llllll}
0.33 & 0.27 & 0.20 & 0.13 & 0.07
\end{array}\right)^{*} \\
\left(\begin{array}{lllllll}
0.23 & 0.06 & 0.20 & 0.20 & 0.36 & 0.04 \\
0.45 & 0.11 & 0.36 & 0.46 & 0.00 & 0.00 \\
0.24 & 0.36 & 018 & 0.24 & 0.43 & 0.23 \\
0.10 & 0.31 & 0.17 & 0.10 & 0.13 & 0.16 \\
0.00 & 0.17 & 0.00 & 0.10 & 0.07 & 0.13
\end{array}\right) \\
=\left(\begin{array}{llllll}
0.24 & 0.17 & 0.23 & 0.27 & 0.23 & 0.15
\end{array}\right)
\end{gathered}
$$

Athlete $S_{2}$ linear transformation is

$$
\begin{gathered}
b_{2}=\left(\begin{array}{llllll}
0.33 & 0.27 & 0.20 & 0.13 & 0.07
\end{array}\right)^{*} \\
\left(\begin{array}{lllllll}
0.03 & 0.51 & 0.20 & 0.30 & 0.40 & 0.07 \\
0.10 & 0.27 & 0.36 & 0.40 & 0.37 & 0.37 \\
0.47 & 0.13 & 018 & 0.17 & 0.00 & 0.40 \\
0.20 & 0.11 & 0.17 & 0.07 & 0.17 & 0.07 \\
0.20 & 0.00 & 0.00 & 0.07 & 0.07 & 0.10
\end{array}\right) \\
=\left(\begin{array}{llllll}
0.17 & 0.28 & 0.29 & 0.24 & 0.26 & 0.22
\end{array}\right)
\end{gathered}
$$

Athlete $S_{3}$ linear transformation is

$$
\begin{gathered}
b_{3}=\left(\begin{array}{llllll}
0.33 & 0.27 & 0.20 & 0.13 & 0.07
\end{array}\right)^{*} \\
\left(\begin{array}{lllllll}
0.00 & 0.03 & 0.00 & 0.30 & 0.10 & 0.07 \\
0.07 & 0.20 & 0.20 & 0.30 & 0.17 & 0.07 \\
0.23 & 0.17 & 0.63 & 0.27 & 0.67 & 0.20 \\
0.17 & 0.57 & 0.13 & 0.07 & 0.03 & 0.57 \\
0.53 & 0.03 & 0.03 & 0.07 & 0.03 & 0.10
\end{array}\right) \\
=\left(\begin{array}{llllll}
0.12 & 0.18 & 0.20 & 0.24 & 0.22 & 0.16
\end{array}\right)
\end{gathered}
$$

We get corresponding set from three competitive aerobics athletes body functions linear transformation as:

$$
b=\left(\begin{array}{lll}
0.24 & 0.17 & 0.12 \\
0.17 & 0.28 & 0.19 \\
0.23 & 0.29 & 0.20 \\
0.27 & 0.24 & 0.24 \\
0.23 & 0.26 & 0.22 \\
0.15 & 0.21 & 0.16
\end{array}\right)
$$

Targeted at competitive aerobics athletes body functions, referencing formers research results, we can get corresponding weights table, and combine with the paper contents, we can get:

$$
\begin{aligned}
& A=\left(a_{11}, a_{22}, a_{33}\right) * b=\left(\begin{array}{llllll}
0.20 & 0.10 & 0.10 & 0.25 & 0.15 & 0.20
\end{array}\right) \\
& \left(\begin{array}{lll}
0.24 & 0.17 & 0.12 \\
0.17 & 0.28 & 0.19 \\
0.23 & 0.29 & 0.20 \\
0.27 & 0.24 & 0.24 \\
0.23 & 0.26 & 0.22 \\
0.15 & 0.21 & 0.16
\end{array}\right) \\
& =\left(\begin{array}{lll}
0.22 & 0.23 & 0.19
\end{array}\right)
\end{aligned}
$$

By above, it is known that three competitive aerobics athletes' body function comprehensive evaluation scores vectors $A_{1}=0.22, A_{2}=0.23, A_{3}=0.19$, then corresponding ranking is : $A_{2}$ is larger than $A_{1}$ is larger than $A_{3}$. So we can get three competitive aerobics athletes body functions sports performances every indicator and individual scores results as Table 2.

By above Table 2, we can know that even competitive aerobics athletes body function $s_{2}$ in 30 seconds push-up is lower than $s_{1}$, but in lateral split index, 30 seconds abdomen contracting and leg raising and else other five aspects are not low, some are even surpassing competitive aerobics athlete body function $S_{1}$, so $S_{2}$ in total competitive aerobics body function performance is superior to $s_{1}$, and $s_{3}$ each item performance is lower than $S_{1}$, so the three competitive aerobics athletes body functions comprehensive ranking is : $s_{1}<s_{3}<s_{2}$.

\section{CONCLUSION}

By making comprehensive evaluation on competitive aerobics athletes body function, it well verifies fuzzy mathematics adaptability and effectiveness, and utilizes performance quantization method, after making comprehensive evaluation on one group of competitive aerobics athletes body functions, obtained result is competitive aerobics athletes body function belongs to good level, to further research, the paper carries out concrete analysis on three competitive aerobics athletes body function, finally gets the three competitive aerobics athletes body function total perform- 
Table 2. Three athletes' sports performances every indicator individual scores.

\begin{tabular}{|c|c|c|c|c|c|c|}
\hline Factor $u_{i}$ & $\begin{array}{c}\text { Lateral Split } \\
\text { Index } u_{1}\end{array}$ & $\begin{array}{c}\text { Vertical Split } \\
\text { Index } u_{2}\end{array}$ & $\begin{array}{c}\text { Standing Long } \\
\text { Jump } u_{3}\end{array}$ & $\begin{array}{c}\text { 30 Seconds } \\
\text { Push-up } u_{4}\end{array}$ & $\begin{array}{c}\text { Cross Jump- } \\
\text { ing Test } u_{5}\end{array}$ & $\begin{array}{c}\text { 30 Seconds Abdomen } \\
\text { Contracting and Leg } \\
\text { Raising } u_{6}\end{array}$ \\
\hline \hline $\begin{array}{c}\text { Weight } a_{i j} \\
\text { Competitive } \\
\text { aerobics athlete } \\
s_{1}\end{array}$ & 0.20 & 0.10 & 0.10 & 0.25 & 0.15 & 0.20 \\
\hline $\begin{array}{c}\text { Competitive } \\
\text { aerobics athlete } \\
s_{2}\end{array}$ & 0.05 & 0.03 & 0.02 & 0.06 & 0.02 & 0.05 \\
\hline $\begin{array}{c}\text { Competitive } \\
\text { aerobics athlete } \\
s_{3}\end{array}$ & 0.04 & 0.02 & 0.03 & 0.04 & 0.03 & 0.22 \\
\hline
\end{tabular}

ances rank $s_{1}<s_{3}<s_{2}$, by above result, we can summarize that future if competitive aerobics athletes want to promote their body functions, they should strengthen their exercises so as to improve their body comprehensive function levels.

\section{CONFLICT OF INTEREST}

The author confirms that this article content has no conflict of interest.

\section{ACKNOWLEDGEMENTS}

This work is supported by the aerobics human institute of science and technology quality course construction projects.

\section{REFERENCES}

[1] L. Qingqing, "Pondering over about the callisthenic teaching of the ordinary colleges and universities", Sports Science Research, vol. $1,1998$.
[2] D. Dong-sheng, "State investigation and countermeasure analysis on aerobics teaching to art major students of the art college", Fujian Sports Science and Technology, vol. 27, no. 1, pp. 51-53, 2008.

[3] Y. Ji-ping, and R. Meng-hua, "A study on the teaching pattern of callisthenics", Journal of Physical Education Institute of Shanxi Teachers University, vol. 18, no. 4, pp. 47-49, 2003.

[4] G. Gai-ling, "Research on the effects of callisthenics on the physical quality of the college girl students", Journal of Physical Education Institute of Shanxi Teachers University, vol. 17, no. 4, pp. 4142, 2002.

[5] L. Jie, and L. Hui-jun, "On the teachers education of callisthenics in colleges and universities", Journal of Liuzhou Teachers College, vol. 20 , no. 4 , pp. 84-85, 2005 .

[6] L. Xiu_hua, L. Cheng, and Y. Xiao_hong, "The reform of callisthenic examination methods in university", Journal of Guangzhou Physical Education Institute, vol. 21, no. 4, pp. 107-109, 2001.

[7] W. Jie-feng, "Problems and solutions: On bilingual teaching in colleges and universities", Journal of Fuyang Teachers College (Social Science Edition), vol. 1, pp. 146-148, 2010.

[8] W. Jinfang, "On the development trend or popular callisthenics", Journal of Hubei Sports Science, vol. 1, 1997.

[9] L. Lin, "On quality education and the improvement of teaching method of areobics dancing", Journal of Guangzhou Physical Education Institute, vol. 21, no, 4, pp. 110-112, 2001.

(C) Liping Wang; Licensee Bentham Open.

This is an open access article licensed under the terms of the (https://creativecommons.org/licenses/by/4.0/legalcode), which permits unrestricted, noncommercial use, distribution and reproduction in any medium, provided the work is properly cited. 